\title{
Patterns in Business Analysis and Enterprise Modeling: How to evaluate their value?
}

\author{
Kurt Sandkuhl \\ The University of Rostock, Albert-Einstein-Str. 22, \\ 18059 Rostock, Germany \\ kurt.sandkuhl@uni-rostock.de
}

\begin{abstract}
Business analysis projects often are considered as activities highly specialized for the specific enterprise under consideration. However, different enterprises in the same industry or service area often show similarities when it comes to organization structures and business processes. This indicates the potential to reuse at least some of the knowledge from earlier business analysis projects in later projects for enterprises of the same sector. Different techniques and approaches have been investigated and proposed for supporting knowledge reuse in analysis and solution design contexts. This paper focuses on the approach to use patterns for this purpose. The major question addressed is the evaluation of the "value" of patterns for the business analysis process and the solution developed. The contributions of the paper are (1) a proposal for how to evaluate the maturity and value of patterns, (2) two actual pattern types and results regarding their evaluation and (3) a discussion of pattern use in the context of business analysis.
\end{abstract}

Keywords: Patterns, Evaluation, Knowledge Reuse, Validation

\section{Introduction}

Business analysis in general is supposed to support enterprises in identifying shortcomings and improvement potential, and in developing solutions to business problems [1]. Solutions potentially encompass different aspects, including strategic planning, policy development, process improvement, organizational change or systems development. Enterprise modeling and business analysis are closely related. Enterprise modeling is providing methods, techniques and tools for capturing "as-is" and "to-be" structures and processes in organizations [2]. Thus, enterprise modeling supports performing business analysis and developing solutions for enterprises.

Business analysis projects often are considered as activities highly specialized for the specific enterprise under consideration, i.e. every enterprise has its own characteristics and needs, which have to be taken into account when setting up a project and which makes every project unique. However, different enterprises in the same industry or service area often show commonalities and similarities when it comes to organization structures and business processes. This indicates the potential 
to reuse at least some of the knowledge from earlier business analysis projects in later projects for enterprises of the same industry or service sector.

Different techniques and approaches have been investigated and proposed for supporting knowledge reuse in analysis and solution design contexts. At a high abstraction level, reference models or frameworks are a recognized approach for capturing knowledge about solution design. Examples for such frameworks are COBIT [3], TOGAF [4] or ITIL [5]. The use of patterns is another accepted approach in this area and addresses a lower level of abstraction than frameworks. When it comes to reusing knowledge about procedures and role distributions in business analysis or solution development projects, various methods have been proposed as means of capturing proven knowledge as support for action. Examples are methods like ARIS [6], OneMethodology, C3S3P [7] or 4EM [8].

In the context of knowledge reuse in business analysis and enterprise modeling, this paper focuses on the approach to use patterns for this purpose. The major question addressed in this work is the evaluation of the "value" of patterns for the business analysis process and the solution developed. Section 2 will give background information to knowledge patterns and to assessing the value of such IT-related artefacts. Section 3 proposes an approach for evaluating maturity and value of patterns, which is derived from experiences in previous enterprise modeling projects. We will use two pattern types for illustrating the approach and our value considerations: task patterns and information demand patterns. Section 4 and 5 will present the two pattern types, which both were developed, applied and evaluated in industrial settings. Section 6 discusses the use of patterns in business analysis. Section 7 draws conclusions and recommends future work.

The main contribution of the paper are (1) a proposal for how to evaluate the maturity and value of patterns, (2) two actual pattern types and results regarding their evaluation and (3) a discussion of pattern use in the context of business analysis.

\section{Background}

Two areas forming the background for the work presented in this paper will be briefly introduced in this section: knowledge patterns (2.1) and approaches for assessing the business value of IT artefacts, like pattern descriptions or models (section 2.2).

\subsection{Capturing Organizational Knowledge with Patterns}

For more than a decade, patterns have been popular in information systems and were introduced for numerous areas, like software design, information modeling or business processes. Although there is no generally accepted definition of the term pattern, most publications in the field get some inspiration from Christopher Alexander's definition, who is an architect and was concerned with buildings: "Each pattern describes a problem which occurs over and over again in our environment, and then describes the core of the solution to that problem, in such a way that you can 
use this solution a million times over, without ever doing it the same way twice" [9]. Whilst Alexander's focus is on the solution, many pattern approaches in information systems focus more on capturing proven practices or an advice on, how to approach certain problems.

In computer science, a number of different pattern types have been proposed. The seminal book on patterns was published by the "Gang of Four" [10] and focuses on software design patterns. Afterwards, patterns basically were proposed for all phases of the software development process, including analysis patterns [11] and software architecture patterns [12]. The term knowledge pattern has been explicitly defined by Clark, Thomson and Porter in the context of knowledge representation [13]. They define "a pattern as a first-order theory whose axioms are not part of the target knowledge-base, but can be incorporated via a renaming of the non-logical symbols" [13, p.6]. The intention is to help construct formal ontologies by explicitly representing recurring patterns of knowledge, so called theory schemata, and by mapping these patterns on domain-specific concepts.

Despite the many different aspects addressed by these different pattern types, they share some characteristics:

- They are based on experiences and deeply rooted in the practice of the field.

- They are not meant to be used blindly as they are. You have to understand the core idea within the pattern and adjust or apply it for the problem at hand.

- They do not only help to build software, processes or models, but also to communicate approaches within a team or between different stakeholders.

Organizational knowledge patterns also show the above characteristics but emphasize more explicitly the focus on organizational knowledge. Organizational knowledge pattern (OKP) are defined as follows [14]: An organizational knowledge pattern is a formalization of knowledge for a recurring organizational task abstracting from organization-specific aspects, which is of value for an organizational actor and an asset for an organization. The pattern examples examined in sections 3 and 4 are OKP and show the following characteristics:

- OKP represent recurring organizational tasks and are at the same time abstracting from a specific organization, i.e. like most other kinds of patterns in computer science is the description of the core elements independent from the actual solution for an organization.

- OKP represent organizational knowledge, not individual knowledge, i.e. support the organizational knowledge management, the organizational context for use of knowledge as opposed to supporting knowledge creation of an individual

- OKP are an asset of the organization, i.e. they are of value for an organizational actor and they are not only a resource as such but capture knowledge about the resource's use.

- OKP are expressed in a formalized way, which requires a formal language or at least a structured representation. At the same time, the cognitive and technical quality of an OKP is adequate for the stakeholders, i.e. the OKP are developed and described for a defined context of usage with identified stakeholders.

It should be noted that there are many more developments in the field of reusable organizational knowledge which are not explicitly called knowledge patterns or 
semantic patterns. Other developments in this area are for example work flow patterns proposed by van der Aalst and colleagues [15], patterns for groupware [16] or patterns of interaction amongst actors [17].

\subsection{Assessing the value of IT-artefacts}

The different pattern types discussed in section 2.1 were applied in many solution development or analysis projects. Although their general benefits have been thoroughly investigated and accepted, the value of this kind of artifact still is subject to research. Numerous research activities from business administration, national economy, computer science and other areas have addressed this area during the last two decades as the "business value of information technology" (BVIT). BVIT can be defined as measures that evaluate how IT-related changes and investments contribute over time to business performance, competitiveness, innovation and economic growth. At least four different types of measurement approaches can be identified:

- Process-oriented approaches (e.g. Mooney et al. IT Business Value Metrics)

- Perceived value approaches (e.g. DeLone and McLean's IS Success Model)

- Project-focused approaches (e.g. Information Economics by Parker)

- multi-perspective approaches based on performance indicators (e.g. Zee's BTriple-E-Framework)

In process oriented approaches the BVIT is demonstrated through process improvements. These approaches answer the question of how value is added to the business. Measures of productivity need to be expanded to capture the impacts of IT use. The organizational context and competitive position should be considered more in studies of business value. Mooney et al. [18] provide a framework that is a basis for process oriented studies of BVIT. They propose a process view for three reasons: first to identify the value adding mechanisms of IT, second to develop an approach and a set of metrics for measuring the technology's value and third to enhance the understanding of the relationship between IT and organizations.

The IS success model by DeLone and McLean [19] belongs to the perceived value approaches. This class of approaches bases BVIT evaluations on user perceptions rather than on financial indicators or measurements within technical systems. DeLone and McLean identified six major dimensions of IS success.

- System quality: Measures of the information processing system itself.

- Information quality: Measures of information system output. The focus is on the quality of the information that the system produces.

- Use: Recipient consumption of the output of an information system.

- User satisfaction: Recipient response to the use of the output of an information system.

- Individual impact: The effect of information on the behavior of the recipient. "Impact" is related to performance, and so improving performance is an indicator that the information system has had a positive impact.

- Organizational impact: the effect of information on organizational performance. 
Information economics by Parker [20] is a methodology for project-justification. It tries to regard the contribution of an IS in terms of business value and seeks to create a rational basis for summarizing complex expressions of value. Representative stakeholders and risk factors influencing the delivery of the business value are identified. The model starts with financial and strategic objectives, and with technical dimensions of the business. An evaluation scale for each category is devised and the business and IS representatives of an enterprise have to negotiate the importance of each evaluation criterion via a weighting factor. This leads to project prioritization and generates an understanding of how IT is adding value to the business (see figure 3).

Zee developed the BTRIPLEE-Framework [21] for IT management and measurement as a "dashboard" of relevant performance indicators for IT. The basic idea is that is no single performance measure to determine the BVIT, therefore multiple measures are needed to reflect different relationships at different levels. An aggregated approach should be applied to correlating IT cost with the performance of the organization. The framework consists of three levels:

- Business Value of IT: IT contributes to business objectives and to business strategy

- Effectiveness of IT: IT effectively supports business processes, activities and employees

- Effectiveness of IT supply: IT supply aligns with business requirements

\section{Pattern validation approach}

For the evaluation of OKP a long term strategy was developed including four different evaluation stages and four different perspectives. The intention was to reach an as high as possible maturity level of the patterns since they were made for industrial use and to collect as many as possible improvement proposals while evaluating the patterns from various perspectives. After having completed all four evaluation stages from four different perspectives, the patterns were expected to be adequate for use both by business analyst and companies developing solutions inhouse.

The evaluation strategy is based on integrating two different lines of work from previous OKP development and evaluation projects. The first line of work focused on pattern use as part of solutions, i.e. the evaluation of the solution was considered, not the elicitation process of the pattern type and the actual pattern. This approach and its use for evaluating task patterns (see section 5) including development methodology are described in [32]. Benefits and shortcomings of task patterns were evaluated from the economic perspective. The balanced scorecard approach [33] was selected as a suitable approach for this purpose, i.e. to structure the objectives for the evaluation process and to implement a measurement system. The second line of work investigated whether evaluation strategies from naturalistic inquiry could be applied for OKP and resulted in an iterative and incremental approach presented in [28]. In this approach, both, pattern type elicitation and actual pattern development were developed. 
The four evaluation stages and perspectives of the proposed long-term strategy are described in the following. The stages include concepts from perceived-value and multi-perspective evaluation discussed in section 2.2. Stage 1 to 3 are from the second line of work; stage 4 is from the first one:

- Assessment of the patterns by experts from the field. This first stage should be started after completion of the work of the pattern development team, i.e. the experts should have been involved in the development work but familiar with the needs of business analysts and companies. After completing this stage, conceptual flaws and obvious shortcomings impeding the use of patterns in practice should have been removed.

- Evaluation by the pattern users. The second stage focuses on the perceived use of the pattern users, which should include both, business analysts and domain experts in companies. The perception of the patterns from the users' perspective is supposed to indicate potentially missing aspects in the pattern and shortcomings in pattern presentation and description.

- Case studies. The third stage requires real-world cases of using patterns for analysis or solution development projects. These cases have to be observed by researchers who document all activities in the project using the case study method. In addition to potential shortcomings in the pattern, success or failure of completing the planned project and the role of patterns in this has to be the main focus of attention in the case study.

- Measurement using scorecards. Whilst case studies mainly focus on collecting qualitative aspects of a case in order to understand interrelations and correlations in one case, measuring effects of pattern use would need many different cases and should include various perspectives which can be represented and captured using indicators. This more long-term way of assessing would require several cases and contexts where solving similar tasks with and without patterns can be observed.

Regarding the perspectives, it is important for the evaluation to distinguish the view of an individual using the patterns from the view of an organization using patterns. Furthermore, the concept of information demand patterns and the actual patterns should be differentiated. This leads to four perspectives:

- the pattern concept from the perspective of an individual

- the pattern concept from the perspective of an organization

- the actual pattern from the perspective of an individual

- the actual pattern from the perspective of an organization

Table 2 illustrates the stages and perspectives, and shows the evaluation steps performed so far for the two pattern types presented in sections 4 and 5. Information demand pattern (IDP) evaluation was completed for the first three stages, task pattern evaluation for stages 1,3 and 4. Task patterns were only evaluated from the organizational perspective. 
Table 2: Evaluation stages and perspectives including the activities performed for the pattern types presented in sections 4 and 5: information demand patterns (IDP) and task patterns (TP)

\begin{tabular}{lllll}
\hline & $\begin{array}{l}\text { Pattern: } \\
\text { Individual } \\
\text { perspective }\end{array}$ & $\begin{array}{l}\text { Pattern: } \\
\text { organizational } \\
\text { perspective }\end{array}$ & $\begin{array}{l}\text { Concept: } \\
\text { Individual } \\
\text { perspective }\end{array}$ & $\begin{array}{l}\text { Concept: } \\
\text { Organizational } \\
\text { perspective }\end{array}$ \\
\hline $\begin{array}{l}\text { Assessment: } \\
\text { Expert Reviews }\end{array}$ & $\begin{array}{l}\text { IDP: } \\
\text { [25] and [26] }\end{array}$ & $\begin{array}{l}\text { IDP: [24] for } \\
\text { collaborative } \\
\text { engineering } \\
\text { TP: [32] }\end{array}$ & $\begin{array}{l}\text { IDP: } \\
\text { [25] and [26] }\end{array}$ & $\begin{array}{l}\text { IDP: [27] for } \\
\text { change } \\
\text { management } \\
\text { TP: [30] }\end{array}$ \\
\hline $\begin{array}{l}\text { Perception: } \\
\begin{array}{l}\text { User } \\
\text { Evaluation }\end{array}\end{array}$ & IDP: subject of section 4.2 & \\
\hline $\begin{array}{l}\text { Observation: } \\
\text { Case Studies }\end{array}$ & IDP: [24] and [27] & IDP: [28 & \\
\hline $\begin{array}{l}\text { Measurement: } \\
\text { Scorecard }\end{array}$ & IDP: Not performed yet & & \\
\hline
\end{tabular}

\section{Information Demand Patterns}

This section introduces the concept of information demand patterns (section 4.1) and selected evaluation results (section 4.2) taken from the user evaluation stage of the approach presented in section 3 .

\subsection{Information Demand Patterns}

The general idea of information demand patterns (IDP) is similar to most pattern developments in computer science: to capture knowledge about proven solutions in order to facilitate reuse of this knowledge. In this paper, the term information demand pattern is defined as follows [22]: An information demand pattern addresses a recurring information flow problem that arises for specific roles and work situations in an enterprise, and presents a conceptual solution to it.

An information demand pattern consists of a number of essential parts used for describing the pattern: pattern name, organisational context, problems addressed, conceptual solution (consisting of information demand, quality criteria and timeline), and effects. These parts will be described in the following. An example for an actual pattern is presented in section 3.2.

- The pattern name usually is the name of the role the pattern addresses. 
- The organisational context explains where the pattern is useful. This context description identifies the application domain or the specific departments or functions in an organisation forming the context for pattern definition.

- The problems of a role are identified. The tasks and responsibilities a certain role has are described in order to identify and discuss the challenges and problems, which this role usually faces in the defined organisational context.

- The conceptual solution describes how to solve the addressed problem. This includes the information demand of the role, which is related to the tasks and responsibilities, a timeline indicating the points in time when the information should be available, and quality criteria for the different elements of the information demand. These criteria include the general importance of the information, the importance of receiving the information completely and with high accuracy, and the importance of timely or real-time information supply.

- The effects that play in using the proposed solution are described. If the needed information should arrive too late or is not available at all, this might affect the possibility of the role to complete its task and responsibilities. Information demand patterns include several kinds of effects: potential economic consequences; time/efficiency effects; effects on increasing or reducing the quality of the work results; effects on the motivation of the role responsible; learning and experience effects; effects from a customer perspective.

The above parts of a pattern are described in much detail in the textual description of the pattern. Additionally, a pattern can also be represented as a visual model, e.g. a kind of enterprise model. This model representation is supposed to support communication with potential users of the pattern and solution development based on the pattern.

Examples of the 27 information demand patterns developed so far include:

- Change administrator in engineering change management,

- Responsible for quote preparation in automotive industries,

- Responsible for material specification in automotive supplier industries,

- Responsible for branding in software industries, or

- Responsible for proposal writing in research organizations. The pattern development process is described in [23].

\subsection{User Evaluation of Information Demand Patterns}

During autumn 2011 and spring 2012, the pattern "Proposal Writing Responsible" was evaluated regarding user perception from all four perspectives introduced in section 3.3, i.e. from individual and organisational perspective for both pattern concept and actual pattern. In spring 2013, this evaluation was extended by including additional participants. The pattern originates from the research project Information Logistics for SME (infoFLOW) [23]. For brevity reasons, we will only present the context and problems addressed in this pattern: 


\section{Context:}

Research and development projects at universities or research institutes to a large extent are based on project funding. Preparing successful proposals for funding is a task that requires experience and accurate information about the published call, funding conditions, economic parameters, etc., which usually originate from different information sources and actors. The pattern describes the information demand typically experienced by the role responsible for preparing a proposal for funding. [...] The pattern is supposed to be useful for any type of organization aiming at submitting proposals for project funding to funding agencies.

\section{Problem:}

The pattern addresses the general problem of submitting proposals of unnecessary low quality, which basically is wasting resources at the preparing organization or reducing the probability and volume of grants. This includes the following problems, which were often observed when preparing proposals for funding:

- Submitted proposals fail to meet the formal requirements published in the call, which usually leads to a rejection. Examples are missing attachments, missing signature, wrong number or different kind of partners.

- Submitted proposals contain an invalid or incorrect budget. Typical mistakes are to include non-eligible costs, use the wrong cost rates or don't include applicable surcharges

- Proposals ask for funding of activities, which already are being funded in other projects of the same fund giver

- Proposals are submitted to late, either due to deadline changes or misunderstandings

- $[\ldots]$

The evaluation was based on two different questionnaires. One was directed towards managers or senior researchers coordinating project acquisition within an organisation. It included in total 15 questions, 9 of them using a 5 point Likert scale, 3 with a "yes/no/don't know" scale and 3 with free form answers. The questions addressed the perceived utility of the different parts of the pattern and the pattern as such. Furthermore, it was investigated whether the organisation under consideration already had trainings or standards for proposal writers implemented. The other questionnaire was towards researchers who either already have experiences in proposal writing or are interested in writing proposals for funding. The questionnaire included 12 questions, 8 of them using a 5 point Likert scale, 2 with a "yes/no/don't know" scale and 2 with free form answers. The focus again was on perceived utility of the pattern as such and different parts of the pattern.

The number of respondents varied for both questionnaires and will be given when presenting the results in the following:

Organisational Perspective. The participants were 5 different organisations from 3 different countries among them 2 research institutes and 3 universities. The participants had the role of Vice-director (2), Assoc. Dean for Research (2), and Head of the Institute. The participating organisations write 25 - 100 proposals / year. In 
proposal preparation, more than 20 employees are involved. Selected results of the survey are that all respondents agree

- "Very much" / "much" that the tasks and responsibilities defined for the role of "Proposal Responsible" are correct and complete,

- "Very much" / "much" with the information demand quality criteria

- that the described effects are valuable / very valuable (exception: timeline, motivation)

- that all elements of the pattern are needed

- The respondents identified a number of potential applications of the patterns, i.e. they could imagine to use the demand pattern for

$\square$ training of new employees

$\square$ Training of current employees

$\square$ Basis or best practice description

$\square$ Improvement of best practices

Individual Perspective. The participants in this part of the survey were from the organizations mentioned above (see a)) and included:

- $3 \mathrm{PhD}$ students (experience: less than 2 proposals)

- 4 researchers (exeperience: 4-6 proposals)

- 1 Head of research group (experience: 10-12 proposals)

- 2 senior researchers (experience: more than 20 proposals)

Selected results of the survey are:

$\square$ All respondents agree "very much" / "much" with the tasks / responsibilities defined for the "Proposal Responsible"

$\square 70 \%$ find that the information demand, quality criteria, effects and task \& responsibility descriptions are "valuable" or "very valuable"

$\square 90 \%$ find that all elements of the pattern are needed

Furthermore, the respondents state that it is difficult to estimate how much effort the overall pattern or the different parts of the information demand pattern would save. Three respondents propose to extend the information demand by an additional element providing "background information from program manager at the fund giver"

Summarizing the results from the survey it can be stated that both the structure for describing information demand patterns and the actual pattern of the "Proposal Responsible" were perceived useful, pertinent and adequate. The most interesting result from our perspective is that this is confirmed from organizational and individual perspective. The biggest shortcoming of the survey is the small number of respondents and the way of selecting the respondents. The authors of this paper had previous contacts to at least one person in all five organizations included in the survey. Even though these "contact persons" did not participate in the survey, the result may be biased due to the pure existence of this previous connection. If the survey is repeated, the participants ideally should be selected randomly and the number of participating organization and individuals should be increased. 


\section{Task Patterns}

The second pattern type discussed is the "task pattern". This section introduces the concept of task patterns (section 5.1) and selected evaluation results (section 5.2) taken from the measurement stage of the approach presented in section 3 .

\subsection{Task Patterns}

The concept of task patterns is a result of the EU-FP6 project MAPPER. In this project, collaborative engineering was supported by adaptable models capturing best practices for reoccurring tasks in networked enterprises. These best practices were represented as active knowledge models using the POPS* perspectives. Active knowledge models are visual models of selected aspects of an enterprise, which cannot only be viewed and analyzed, but also executed and adapted during execution. The POPS* perspectives include the enterprise's processes $(\mathrm{P})$, the organization structure $(\mathrm{O})$, the product developed $(\mathrm{P})$, the IT system used $(\mathrm{S})$ and other aspects deemed relevant when modeling $(*)$ [29].

The term "task patterns" was introduced for these adaptable visual models, as they are not only applicable in a specific company, but are also considered relevant for other enterprises in the application domain under consideration. Task pattern in this context is defined as "self-contained model template with well-defined connectors to application environments capturing knowledge about best practices for a clearly defined task" [30]. In this context, self-contained means that a task pattern includes all POPS* perspectives, model elements and relationships between the model elements required for capturing the knowledge reflecting a best practice. Model template indicates the use of a well-defined modeling language and that no instances are contained in the task patterns. Connectors are model elements representing the adaptation of the task pattern to target application environments.

Reusing organizational knowledge will in practical contexts require a way to store the pattern and retrieve it for a given problem. This requires a representation suitable for use in knowledge repositories or portals. The representation of a task pattern consists of three main elements:

1. description of the problem addressed by the task pattern. Currently, scenario descriptions represent this part.

2. knowledge model proposing a solution for the problem addressed

3. rationale behind the solution, i.e. an explanation about the most important preconditions, principal results and most important work steps. These elements all are included in the model. The rationale is meant as a support for finding and selecting the best suitable task pattern for a problem.

Examples of the 17 task patterns developed so far include:

- establish material specification,

- establish product specification,

- develop test method,

- perform external testing, or

- target setting. 
All patterns originate from automotive supplier or electrical engineering industries. Pattern examples and more information about the development process can be found in [31] and [32].

\subsection{Scorecard-based Evaluation of Task Patterns}

This section summarizes the evaluation of task patterns from an economic perspective, which was performed using a balanced scorecard and focuses on business value and business drivers like reduced lifecycle time or increased flexibility. The scorecard which was used for the evaluation was newly developed for this purpose (cf. [32]) and included three perspectives with explicitly defined focus and goal. The process perspective focused on the work processes in a product development project. Main attention is put on effects of the adaptability and reconfigurability, which is offered by the model-basing of task patterns. The goal when using the task patterns was high quality and adaptability of the best practice captured in task patterns. The finance perspective aimed at observing potentials effects on the costs related to product design projects. The focus here was on collaboration cost. Main goal was to reduce time and cost for collaboratively performed tasks. The knowledge perspective reflected the objective of supporting distributed groups of engineers in creating innovations. Main focus was on capturing effects of sharing. The goal was intensive knowledge sharing for creating innovations and avoiding errors.

All three perspectives were refined with sub-goals and implemented in a measurement system at four industrial use case partners in the MAPPER project. These partners captured the indicators continuously during a period of one year. The indicators were evaluated at three points in time: at the beginning of the one year period (baseline indicators without using task patterns), in the middle of the period (intermediary indicators) and at the end (final indicators).

In total, the values of 48 indictors were collected at 3 points in time (baseline, intermediary, final), most of them by all four use case partners. From this data, the indicators most relevant for judging the effects of task pattern use in product development were selected and will be discussed in the following. The four use case companies will be referred to as $\mathrm{C} 1, \mathrm{C} 2, \mathrm{C} 3$ and $\mathrm{C} 4$. The development projects performed at these use case partners during measurement of the indicators included a new product version for a seat heating product, a new system-on-chip product and part of the target setting for a new automotive product.

The number of best practice descriptions increased significantly. $\mathrm{C} 1$ reported 7 additional best practice descriptions; C3 reported 5; C2 6 and C4 9. These best practice descriptions are actually the task patterns developed in MAPPER. The overall tendency is clearly positive, as the growing number of task patterns, which are reusable organisational knowledge models, is considered very valuable from the use case partners' perspective. Several indicators were used to contribute to the criteria "quality of best practices", which addressed existing descriptions before the start of MAPPER and the task patterns developed during MAPPER. The average level of detail of the best practice descriptions at $\mathrm{C} 3, \mathrm{C} 1$ and $\mathrm{C} 2$ did not change during the 
runtime of the project. At $\mathrm{C} 4$ the level of detail for the best practices which are part of the use case increased significantly, which is a very positive development.

Furthermore, $\mathrm{C} 4$ investigated whether the best practice descriptions could be used for training new employees or employees who shifted to another role within $\mathrm{C} 4$. The indicator shows that in total 4 employees were trained based on the task pattern, which $\mathrm{C} 4$ considers very successful. From a MAPPER perspective this indicates a clear benefit of MAPPER results.

The criterion "quality of product documentation" included indicators regarding the product structure and regarding design rules. With respect to design rules, $\mathrm{C} 2$ shows a clear increase of the number of rules from 95 to 160; C3 reports an increase from 25 to 27; C4 reports no changes. In all three companies, all existing design rules are in use. The product structure related indicators were only captured by $\mathrm{C} 4$ and show a very positive development. The number of configurable components, which are included in the product structure, increased with the use of MAPPER technology from 0 to 16; the refinement level improved from 3 refinements to 4 ; the number of elements in the product structure remained stable.

$\mathrm{C} 4$ provided indicators, which were contributing to the objective of reducing cycle times and time to market. The criteria connected to this objective were the average length of the development process and of certain phases in this process. The values provided by $\mathrm{C} 4$ are estimations based on the experiences when using the MAPPER infrastructure and based on the expected effect on the overall development process and its phases. A clear reduction of the time needed is reported for the material specification task: from 4 months before MAPPER to 3 months when using MAPPER technologies and methodologies. This reduction by $25 \%$ is considered as a major success story of MAPPER. For the overall process length, no changes are reported. The main reason for this unchanged situation is that the most intense use of MAPPER happened in the material specification task. A complete development project based on MAPPER technology was so far not performed.

\section{Discussion: Pattern Use in Business Analysis}

The two pattern types and their evaluation presented in section 3 and 4 show how perceived value approaches and multi-dimensional approaches for assessing the business value of IT can be applied for evaluating organizational knowledge patterns. Furthermore, the evaluation results indicate that both pattern types provide an actual value to their users. What still needs to be discussed is in what phases patterns could be applied. The presented pattern types were developed with an enterprise modeling perspective in mind. What is the application potential of these patterns from a business analysis perspective?

The Business Analysis Body of Knowledge [1] identifies different knowledge areas which will be used in the following to discuss, in which areas the use of patterns is promising. These knowledge areas are: 
- Business Analysis Planning and Monitoring: identify and plan activities necessary to perform a business analysis and monitor it, including stakeholder analysis, selection of analysis techniques and approach for requirements management,

- Enterprise Analysis: identify business needs and solution approach, define solution scope and business case,

- Elicitation: work with stakeholders to correctly and completely understand their needs,

- Requirements Analysis: elaborate solution definition based on the requirements. This includes an assessment of the current state of the business and identify the need for improvements

- Solution Assessment and Validation: assess proposed solutions to determine the best solution to the business need,

- Requirements Management and Communication: management of conflicts, issues and changes within the business analysis project including communication with the stakeholders and in the project team

The patterns discussed in this paper do not focus on methodological aspects of how to perform certain activities in a business analysis but rather address elements of future solutions to business needs. Thus, knowledge areas with an emphasis on managing project scope, adequate organization structures and communication within the project, e.g. business analysis planning and monitoring, elicitation, requirements analysis, and requirements management and communication, will probably not benefit from pattern use. The following discussion will focus on the solution-related knowledge areas.

In enterprise analysis, patterns can be used to identify the potential source for shortcomings and operational problems in an enterprise. Organizational knowledge patterns, like information demand patterns, offer the possibility to compare proven best practice solutions with the as-is situation in an enterprise which might also contribute to understanding the business needs. However, these patterns do not necessarily indicate the actual solution for the shortcoming detected, since they show information needs which might not be fulfilled, but do not include how the missing information should be provided.

When it comes to defining solutions for the identified business needs, pattern types like task patterns can contribute. This pattern type proposes a solution to a welldefined organizational task, which has to be configured or instantiated for the company under consideration. Since the pattern provides "to-be" processes including the required organizational roles and their competences, resources to be available and the inter-relationship between these perspectives, substantial parts of the solution are pre-defined.

In solution assessment and validation, organizational knowledge patterns can be used as a best practice reference, i.e. the solution developed to the business needs can be compared with the best practice in order to identify differences and potential improvement potential. However, this use of patterns only makes sense if the solution was developed without applying them. 


\section{Summary and Future Work}

The ambition of this paper was to contribute to the evaluation of the "value" of patterns for the business analysis process and for solution development. Using two concrete examples, we showed how evaluation of patterns can be performed and that at least the two presented patterns types have to be considered of value for the analysis activity and solution development in the industrial settings they were developed for. The pattern validation approach introduced in section 3 proposes a way to integrate different validation and evaluation approaches. Experiences from applying this approach for information demand and task patterns indicate that it makes sense to include all four stages, since the maturity of the pattern seems to improve from stage to stage, and that the four perspectives are needed if the pattern type under consideration is supposed to be used by individuals and for organizational purposes. From a scientific perspective, a comparison of the approach with other strategies still has to be performed and using the approach for other pattern types is expected to increase maturity of the approach. Currently, the approach probably has to be considered a "good practice" for validating OKP.

Regarding the usefulness fur business analysis and enterprise modeling in general, the two pattern examples and their evaluation provide only a very limited view, which confirms that there are patterns of organizational knowledge which are useful but not that such patterns are useful in general. However, the way of developing, constructing and evaluating patterns applied in the two example cases might provide a good practice for future pattern types. The discussion in section 6 about utility of patterns for different knowledge areas and phases only provides a first opinion and needs more work in the future.

\section{References}

1. IIBA Business Analysis Body of Knowledge, Version 2.0 Framework. International Institute of Business Analysis. Aviable at http://parisfr.theiiba.orh/download/babok20overview.pdf. Retrieved 2012-08-26 (2012)

2. Vernadat, F.B.: Enterprise Modelling and Integration. Chapman \& Hall (1996)

3. Office of Government Commerce The Official Introduction to the ITIL Service Lifecycle, 2007th ed. London: The Stationery Office (2007)

4. The Open Group TOGAF. Aviable at http://www.opengroup/togaf (2013) Retrieved: 201306-11.

5. IT Governance Institute COBIT 4.1: Framework, control objectives, management guidelines, maturity models. IT Governance Institute, Rolling Meadows, IL (2007)

6. Scheer, A.-W.: ARIS - Modellierungsmethoden, Metamodelle, Anwendungen. 3. Auflage. Berlin, Heidelberg: Springer Verlag (1998) http://dx.doi.org/10.1007/978-3-642-97731-2

7. Sandkuhl, K., Lillehagen, F.: The Early Phases of Enterprise Knowledge Modeling. IFIP PoEM 2008, Springer LNBIP 15, Stockholm, Sweden (2008)

8. Sandkuhl, K., Wißotzki, M., Stirna J.: Unternehmensmodellierung - Grundlagen, Methode und Praktiken. Springer Verlag (2013)

9. Alexander, C. et al.: A Pattern Language. Oxford (1977)

10.Gamma, E., Helm, R., Johnson, R., Vlissides J.: Design Patterns: Elements of Reusable 
Object-Oriented Software, Addison-Wesley (1994)

11.Fowler, M.: Analysis Patterns, Addison Wesley (1997)

12.Buschmann et al.: Pattern-oriented Software Architecture, Wiley (2000)

13.Clark, P., Thompson, J.,Porter B.: Knowledge Patterns. In A. G. Cohn, F. Giunchiglia, and B. Selman, editors, KR2000: Principles of Knowledge Representation and Reasoning, San Francisco, 2000. Morgan Kaufman (2000).

14.Sandkuhl, K.: Organizational Knowledge Patterns: Foundation and Application Example. In: L. Villa-Vargas, L. Sheremetov, and H.-D. Haasis: Operations Research and Data Mining ORADM 2012 workshop proceedings. March 2012, Cancun, Mexico. Mexican Petroleum Institute, Mexico City, 978-607-414-284-6 (2012)

15.Van der Aalst, W.M.P., ter Hofstede, A.H.M., Kiepuszewski, B., Barros, A.P.: Workflow Patterns. Distributed and Parallel Databases, 14, 5-51, Kluwer (2003)

16.Schümmer, T., Lukosch, S.: Patterns for Computer-Mediated Interaction, Wiley \& Sons, ISBN: 978-0-470-02561-1 (2007)

17.Martin, D.: Patterns Of Cooperative Interaction - A Brief Introduction To The Lancaster Perspective. From Good Practices to Patterns. Workshop on mining socio-technical patterns from experience with groupware. ECSCW 2003, Helsinki, Finland (2003)

18.Mooney, J., Gurbaxani, V., Kraemer, K. L.: A Process Oriented Framework for Assessing the business value of information technology. In: Proceedings of the 16th International Conference on Information Systems, Amsterdam, pp.17-27 (1995)

19.DeLone, W., McLean, E.: Information system success: the quest for the dependent variable. Information Systems Research, Vol. 3(1), pp. 60-95 (1992) http://dx.doi.org/10.1287/isre.3.1.60

20.Parker, M., Benson, R.: Information Economics, Prentice-Hall, Englewood Cliffs, NJ (1988)

21.Zee, Han von der: Measuring the Value of Information Technology. Idea Group Publishing, ISBN 1930708084 (2002)

22.Sandkuhl, K.: Information Demand Patterns. Proc. PATTERNS 2011, The Third International Conferences on Pervasive Patterns and Applications, pp. 1-6. September 2530, 2011; Rome, Italy. ISBN: 978-1-61208-158-8 (2011)

23.Lundqvist, M., Sandkuhl, K., Seigerroth, U.: Modelling Information Demand in an Enterprise Context: Method, Notation and Lessons Learned. International Journal Systems Modeling and Design, Vol. 2(3), IGI Publishing, pp. 74-96 (2011)

24.Sandkuhl, K.: Supporting Collaborative Engineering with Information Supply Patterns. Proceedings of $10^{\text {th }}$ Euromicro PDP 2010, Pisa, Italy, IEEE CS (2010)

25.Sandkuhl, K.: Validation and Use of Information Demand Patterns in Higher Education. BIS 2010, Berlin, Germany, May 2010. LNBIP vol. 57, Springer (2010)

26.Sandkuhl, K., Stamer D.: Towards Improving Structure and Content of Information Demand Patterns. In: N. Aseeva, E. Babkin and O. Kozyrev (Eds.): Perspectives in Business Informatics Research - 11th International Conference, BIR 2012, Nizhny Novgorod, Russia, September 24-26, 2012. ISBN 978-5-502-00042-0 (2012)

27.Sandkuhl, K.: Improving Engineering Change Management with Information Demand Patterns. Pre-Proceedings of the IFIP WG 5.1 8th International Conference on Product Lifecycle Management, Eindhoven (NL). Inderscience Enterprises Ltd. (2011)

28.Sandkuhl, K.: Validating Organizational Knowledge Patterns: Case Study from Information Demand Modeling. In: Grabis, J. and Kirikova, M. (eds.) Perspectives in Business Informatics Research. LNBIP 90, pp. 204-216. Springer Verlag (2011) http://dx.doi.org/10.1007/978-3-642-24511-4_16

29.Lillehagen, F.: The Foundations of AKM Technology, Proceedings 10th International Conference on Concurrent Engineering (CE) Conference, Madeira, Portugal (2003)

30.Sandkuhl, K., Smirnov, A., Shilov, N.: Configuration of Automotive Collaborative Engineering and Flexible Supply Networks. In Cunningham, P. and Cunningham, M. (Eds.): Expanding the Knowledge Econom. ISBN 978-1-58603-801-4 (2007)

31.Johnsen, S., Schümmer, T., Haake, J., Pawlak, A., Jørgensen, H., Sandkuhl, K., Stirna, J., 
Tellioglu, H., Jaccuci, G.: Model-based Adaptive Product and Process Engineering. In: Rabe, M.; Mihók, P. (Eds) New Technologies for the Intelligent Design and Operation of Manufacturing Networks. Fraunhofer IRB Verlag, Stuttgart, Germany (2007)

32.Sandkuhl, K.: Capturing Product Development Knowledge with Task Patterns: Evaluation of Economic Effects. Quarterly Journal of Control \& Cybernetics, Issue 1, 2010. Systems Research Institute, Polish Academy of Sciences (2010)

33.Kaplan, R. and Norton, D.: Balanced Scorecard, Harvard Business School Press, Boston, USA (1996) 